\title{
Innovative Energy-Saving Propulsion System for Low-Speed Biomimetic Underwater Vehicles
}

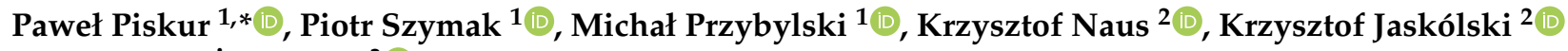 \\ and Mariusz Żokowski ${ }^{3}$ (D) \\ 1 Faculty of Mechanical and Electrical Engineering, Polish Naval Academy, Smidowicza 69, \\ 81-127 Gdynia, Poland; p.szymak@amw.gdynia.pl (P.S.); m.przybylski@amw.gdynia.pl (M.P.) \\ 2 Department of Navigation, Polish Naval Academy, Smidowicza 69, 81-127 Gdynia, Poland; \\ k.naus@amw.gdynia.pl (K.N.); k.jaskolski@amw.gdynia.pl (K.J.) \\ 3 Air Force Institute of Technology, Ks. Boleslawa 6, 01-494 Warsaw, Poland; mariusz.zokowski@itwl.pl \\ * Correspondence: p.piskur@amw.gdynia.pl
}

Citation: Piskur, P.; Szymak, P.; Przybylski, M.; Jaskólski, K.;

Żokowski, M. Innovative

Energy-Saving Propulsion System for Low-Speed Biomimetic Underwater Vehicles. Energies 2021, 14, 8418 . https://doi.org/10.3390/en14248418

Academic Editor: Daniel Chindamo

Received: 19 October 2021

Accepted: 8 December 2021

Published: 14 December 2021

Publisher's Note: MDPI stays neutral with regard to jurisdictional claims in published maps and institutional affiliations.

Copyright: (c) 2021 by the authors. Licensee MDPI, Basel, Switzerland. This article is an open access article distributed under the terms and conditions of the Creative Commons Attribution (CC BY) license (https:/ / creativecommons.org/licenses/by/ $4.0 /)$.

\begin{abstract}
This article covers research on an innovative propulsion system design for a Biomimetic Unmanned Underwater Vehicle (BUUV) operating at low speeds. The experiment was conducted on a laboratory test water tunnel equipped with specialised sensor equipment to assess the FluidStructure Interaction (FSI) and energy consumption of two different types of propulsion systems. The experimental data contrast the undulating with the drag-based propulsion system. The additional joint in the drag-based propulsion system is intended to increase thrust and decrease energy input. The tests were conducted at a variety of fins oscillation frequencies and fluid velocities. The experiments demonstrate that, in the region of low-speed forward movement, the efficiency of the propulsion system with the additional joint is greater.
\end{abstract}

Keywords: Biomimetic Unmanned Underwater Vehicle (BUUV); fish-like movement; underwater robotics; energy efficiency; Fluid-Structure Interaction (FSI); undulating propulsion

\section{Introduction}

Biomimetic propulsion systems are based on biological organisms that evolved through natural selection over many years [1]. In contrast to the screw-type propeller system, biomimetic systems move like a fish [2], a turtle [3], a seal [4] or other marine animal. The undulatory movement of body bending generates propulsion by pushing the water against a passing wave [5]. Due to the benefits of a low hydroacoustic noise spectrum [6], this type of propulsion is becoming more popular in underwater robots $[7,8]$.

However, the influence of tail oscillation on the fluid flow around a hull causes vortices and the need to carefully study the FSI $[9,10]$ and the vortex structures [11]. The underwater vehicles shown in $[7,12]$ have propulsion systems made of several moving parts. For example, the artificial fish in Figure 1a is shown with a hull formed by the coupling of rigid movable parts with a flexible fin [13] at the end. This type of construction is difficult to control, expensive and increases the risk of flooding the electronic components inside.

That is why an effort was made to design a biomimetic propulsion system based on single, flexible fins [14]. The BUUV with a propulsion system consisting of one flexible tail fin and two side fins is depicted in Figure $1 \mathrm{~b}$. This kind of propulsion made it challenging to maintain the BUUV's direction. Furthermore, if hydroacoustic sensors are to be employed, movement stabilization is highly desirable. To increase the course stability and reduce the scrolling impact [4], replaced one tail fin with two tail fins (Figure 1c). 


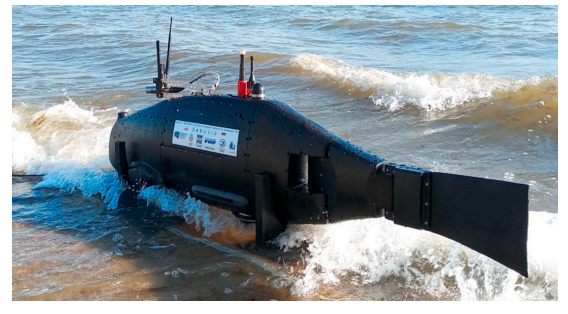

(a)

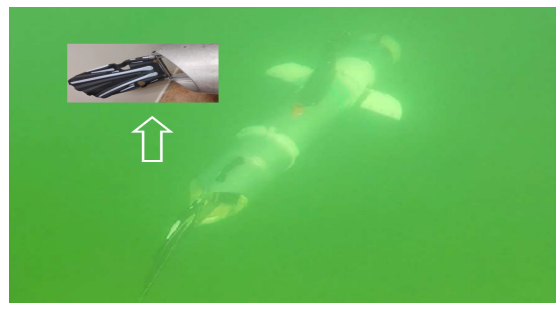

(b)

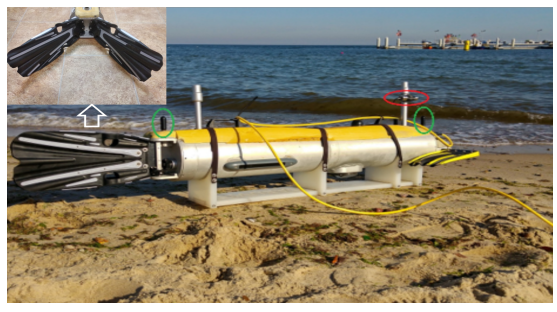

(c)

Figure 1. BUUV with a propulsion system made of (a) movable, rigid elements and a flexible fin [6]; (b) one flexible tail fin [15]; and (c) two flexible tail fins [16].

A proposed innovative propulsion system was a modified version of the two-tail fin propulsion system [4] with reduced drag during the return stroke due to the use of an additional joint. The propulsion design with the additional joint is distinct from prior designs [17-20] particularly during the return movement. Under the effect of fluid drag force, the fin is freely rotated up to 90 degrees during the return stroke.

Although, the authors' paper [21] offered a simulation analysis of a fin drag force in a BUUV using the LS-DYNA software and Incompressible Computational Fluid Dynamics (IFCD) with validation in a water tunnel, in this paper, data are provided based on the measurements made in the water tunnel. This is mainly due to the fact that the thrust and the drag of fins depend on their flexibility and shape for different fluid velocities as well as different frequencies and different amplitudes of oscillation.

The single piece of the fish-like flapping foils and one flipper with an additional joint were compared because they can be used on both sides of the vehicle (pectoral fins) as well as at the aft of the BUUV (caudal fins). The fin system in tandem is to be compared in a later study, and, in that case, the paper concentrates on the mechanism's dynamic of the propulsion system based on one fin. The results were chosen to present the differences between two kinds of propulsion system that can be implemented in the BUUV. The presented differences should support choosing the adequate type of propulsion system depending on the BUUV requirements.

The efficiency comparison between two types of propulsion system is provided according to the research and the analysis presented in the papers [22-24], where defined efficiencies take the kinematic and performance parameters for various measurement conditions into consideration.

The next section includes a description of the new propulsion system. Then, the mathematical analysis of the propulsion system with a discussion on energy efficiency is provided. Further, the results of measurements for the new propulsion system with additional joints to the fish-like flapping foils are compared. At the end of this study, the findings are discussed, and our conclusions as well as future research directions are proposed.

\section{Description of the New Propulsion System with Additional Joint}

A photo sequence is shown in Figure 2 to help describe the new drive mechanism. The two fins mounted on the stern to the vertical rotary axes are tilted synchronously in a horizontal plane by electric motors to ensure the underwater vehicle's translational movement. In the power stroke, the BUUV moves the stern fins from outside to the back to push the water in the opposite direction to the hull movement (Figure $2 b-d$ ). In the return stroke, the BUUV moves the stern fins to the start position, with the drag force reduction provided by the additional joint (Figure 2e-g).

The movement of both fins outwards is simply an auxiliary movement that allows the specified values of the maximum angular deflection of the fins regarding the longitudinal axis of symmetry to be obtained, which is required to begin the work movement. During the movement, the fins alter their position from the vertical to the horizontal plane. This reduces the hydrodynamic drag of the fins during outside movement and, as a result, the drag of underwater vehicles. 
The main benefit of the auxiliary joint is a seamless quarter-turn to a position with the least amount of drag. The next benefit of the proposed concept is that the auxiliary joint does not require a separate servo motor [17].

The next part of the paper provides a more extensive analysis that excludes the impact of the hull on the propulsion system parameters. The one-fin propulsion system is tested to exclude the interaction between both fins. According to former tests [4] with two flexible tail fins (see Figure 1c), that interaction appears to increase the thrust, but herein the number of variables was reduced, and only a one-fin propulsion system is depicted.
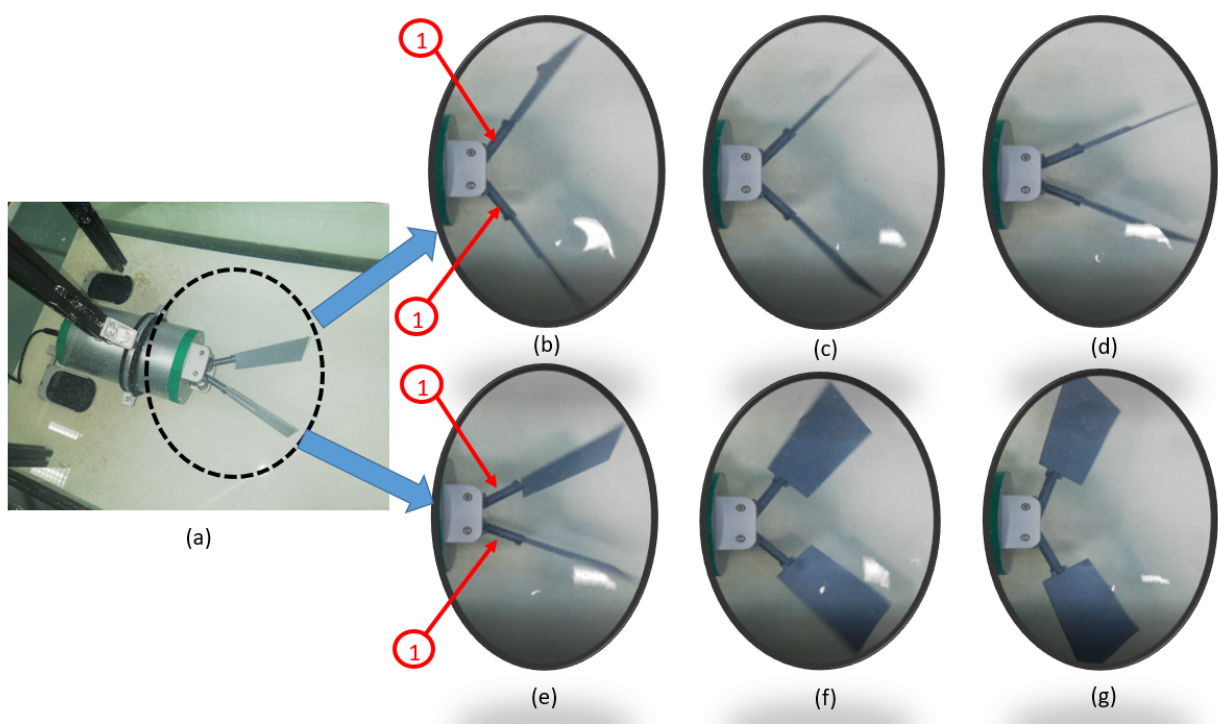

Figure 2. A BUUV (a) with subsequent phases of movement: (b-d) power stroke and (e-g) return stroke; 1 -additional joint.

\section{Mathematical Relations}

The FSI depends on the environmental parameters (describing the fluid properties), geometric parameters (describing the shape of the fins), kinematic parameters (describing the motion of the fins) and performance parameters (describing the propulsion and energy harvesting performance of the fins) [25]. The analysis can be carried out following the Buckingham theory [26]. The principle enables the expression of all the information contained in the problem's interactions between physical variables in a compact manner, employing a reduced number of dimensionless variables. According to the literature review $[25,27]$ and experimental results, the expression characterizing the FSI can be described as follows:

$$
F=\mathbf{f}\left(K, \alpha, S, w, f, \rho, \mu, u_{\infty}\right)
$$

where

$K$ is a geometric parameter that determines the shape of the fin;

$\alpha$ is the angle of attack, measured between the fin chord and the fluid velocity;

$S$ is the fin side area;

$w$ is the fin thickness;

$f$ is the fin movement frequency;

$\rho$ is the fluid density;

$\mu$ is the fluid viscosity; and

$u_{\infty}$ is the free stream fluid velocity.

For incompressible flow, the fluid density $\rho$ and viscosity $\mu$ are considered to be constant. Furthermore, no initial conditions are required if the problem is considered to be steady. If a flexible fin material is used, the shape of a fin constantly varies with its peak-topeak amplitude motion [27]. According to the Buckingham theory, there are eight variables, 
two of which are independent $(K, \alpha)$, and six of which are dependent. The fundamental units are meter, second and kilogram. Consequently, the FSI can be described as follows:

$$
F=\mathbf{f}(K, \alpha, S t, R e, k)
$$

where

St is the Strouhal [28] number depicted in Equation (3);

$R e$ is the Reynolds [29] number, here defined as a ratio of fluid inertia to viscous forces (Equation (4)); and

$k$ is the stiffness coefficient [30] (Equation (5));

$$
\begin{aligned}
& S t=f \frac{A}{u_{\infty}} \\
& R e=\frac{c u_{\infty}}{\mu} \\
& k=\frac{E I}{\rho u_{\infty}^{2} c^{3}}
\end{aligned}
$$

where

$A$ is the peak-to-peak amplitude measured at the fin trailing edge;

$c$ is the chord length of the fin at the midline;

$I=\frac{h^{3}}{12}$ is the area moment of inertia of the cross section; and

$E$ is Young's modulus.

Although the research was provided on the stiffness coefficient [31], it was assumed that the optimal stiffness for fish-like flapping foils is different than for the new design of propulsion system. That is why the new propulsion system with an additional joint was designed as inflexible. This was mainly introduced to decrease the number of variables that have an impact on the propulsion system parameters. The flexible fin with an additional joint is provided in the next step of the research, and then we analyze the impact of flexibility on the propulsion efficiency .

\section{Energy Efficiency}

The energy efficiency of the propulsion system [22,32] is defined as the ratio of useful power output to the rate of power input measured over a specific time interval $[33,34]$.

$$
\eta=\frac{u T_{n}}{P_{f}}
$$

where

$\eta$ is the energy efficiency;

$T_{n}$ is the net thrust; and

$P_{f}$ is the value of electrical power consumed by the servomotor.

In Figure 3 , the two $P_{s}$ and $P_{f}$ are the power consumed by the servomotor when driving without the fin and when driving the fin in water, respectively. The data reported here were obtained by experimentation on the tested fin. The test provided for the frequency of the fin oscillation in the range from 1 to $7.6 \mathrm{~Hz}$.

The power input to the servomotor [35] is determined as a product of the measured voltages $(U)$ and the electric current $(I)[36]$ across the servomotor using the following Equation (7):

$$
P=I U
$$




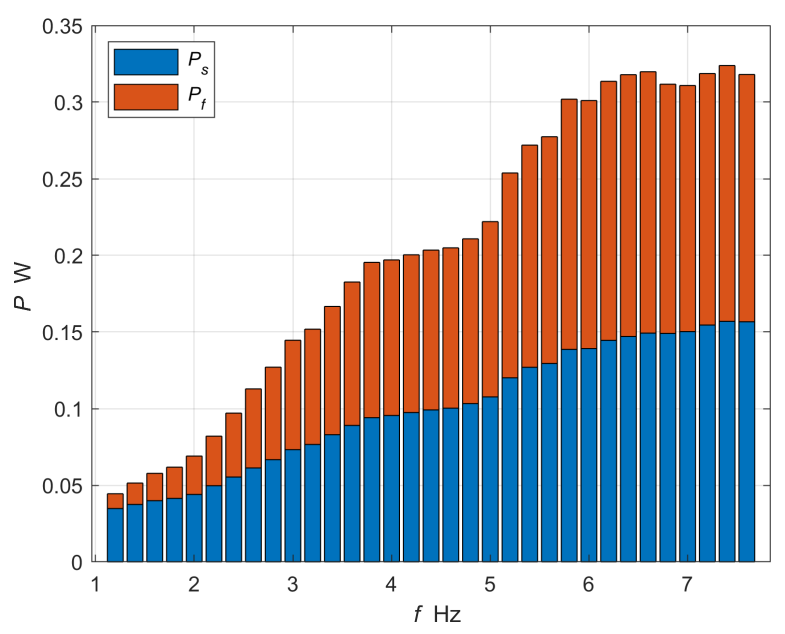

Figure 3. Electric power delivered to the servomotor: $P_{S}$ - power for servomotor without a fin, and $P_{f}$ - power for a fin submerged in the water.

For very low Reynolds numbers, viscous forces produce both the drag and the thrust forces. Furthermore, propulsion forces generated by fin deformation or flipper position and velocity influence the drag forces; for the reason presented in Equation (6), the net thrust defines the difference between thrust and drag force (8).

$$
T_{n}=T+T_{D}
$$

where

$T$ is the thrust; and

$T_{D}$ is the drag force.

For a propulsion system submerged in a viscous fluid, the propeller thrust needs to overcome the resistance force to ensure a constant speed. A steady motion for $T_{n}=0$ means that the propulsion system does not decelerate or produce thrust. For the innovative propulsion system, the net thrust (9) during the working and returning movement of a fin depends on the coefficient $\left(C_{f}\right)$, the side area of the fin $(S)$, both angles $\alpha$ and $\beta$, the square of the fluid velocity $(u)$ and acceleration concerning the fin as well as an added mass $(m)$.

$$
T_{n}(\alpha, \beta)=m(\alpha, \beta) \frac{d u}{d t}+\frac{1}{2} \rho S(\alpha, \beta) C_{f}(\alpha, \beta) u^{2}
$$

where

$\alpha$ is the angle of attack in the horizontal plane;

$\beta$ is the angle of attack in the vertical plane;

$m$ is an added mass; and

$C_{f}(\alpha, \beta)$ is measured experimentally in the laboratory water tunnel [21,37].

The net thrust $\left(T_{n}\right)$ is affected by the position of the fin and the velocity of the water. For $\alpha=90^{\circ}$ and $\beta=0^{\circ}$, the fin is in a neutral position parallel to the vehicle's longitudinal axis, whereas $\alpha=0^{\circ}$ and $\beta=90^{\circ}$ indicate that the fin is entirely deflected in an outward position.

An impulsive motion in water can generate considerable thrust proportional to the fin acceleration. The actual thrust can be difficult to assess accurately because it is difficult to estimate precisely the volume of fluid that is accelerated by a particular motion. Although for simple motions and shapes, some reasonable simulation estimates can be given, here, experiments in the water tunnel were provided.

\section{Laboratory Test Equipment and Measurement Methods}

In [38], a wide range of real caudal fin shape species were digitized and analyzed, ranging from homocercal tails with a low aspect ratio (square shape used by bluegill sunfish 
and rainbow trout) to high aspect ratio (lunate shape adopted by tuna and swordfish) and even the heterocercal caudal fin adopted by sharks.

The comparison of propulsive efficiency shows that large aspect ratio fins (tuna and shark) are more efficient while cruising $(\mathrm{St}<0.4)$, whereas short aspect ratio caudal fins (catfish and trout) are more efficient when fish are accelerating or maneuvering (St $>0.4)$. Given that vortex morphologies are primarily reliant on the fin aspect ratio and trailing edge shape [39], the rectangular shape of the fins was chosen for the study and to compare the efficiency changes caused by the addition of a joint.

In Figure 4, two fins with the same length $(70 \mathrm{~mm})$ and height $(40 \mathrm{~mm})$ are displayed. The variable $M$ indicates the torque from the servomechanism while the variable $u$ indicates the BUUV velocity relative to the water. The fish-like flapping foils (Figure 4a,b) made from flexible, thin steel sheet and flexible polycarbonate were chosen according to earlier experimental testing [31]. The flexibility parameter was selected by changing the thickness of the fin. Due to the needed flexibility and fatigue strength [40], the polycarbonate fin had a width of $0.2 \mathrm{~mm}$, whereas the steel fin had a width of $0.1 \mathrm{~mm}$.

In Figure $4 c, d$, the fin with the additional joint is presented for power (c) and return (d) stroke. The vertical position of the inflexible fin with an additional joint (Figure 4c) generates thrust, while the horizontal position (Figure $4 \mathrm{~d}$ ) is used to reduce the resistance on the return stroke.

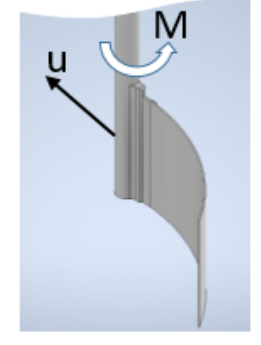

(a)

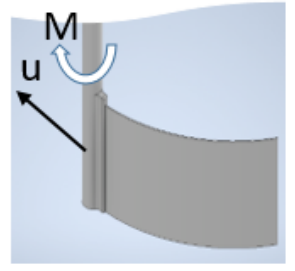

(b)

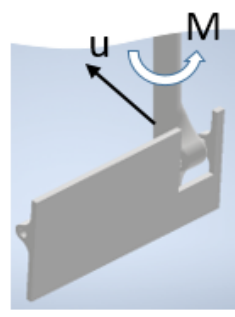

(c)

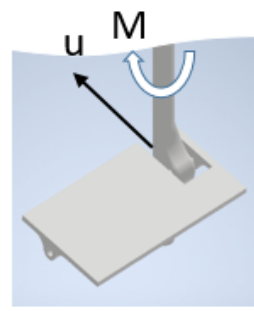

(d)

Figure 4. Two types of propulsion systems used for tests: the flexible fin during counterclockwise (a) and clockwise (b) movement; the flipper with an additional joint during the power (c) and return (d) stroke.

The laboratory test water ring shown in Figure 5 was designed for the characteristic dynamic establishment of the singular fin without considering the effect of the underwater vehicle body. The laboratory water tunnel was composed of polyethylene panels with the following dimensions: $0.09 \mathrm{~m}$ height, $0.22 \mathrm{~m}$ width and $0.02 \mathrm{~m}^{2}$ cross-sectional area. The laboratory test determines the fin dimensions to use as well as the sensors employed.

For example, an overly large fin may interact with the inner sides of the tunnel, while an overly small fin may generate insufficient force to be monitored by strain gauges. The corners were set to $45^{\circ}$ due to laminar flow restrictions, and an additional steering wheel was installed before the place of measurement. An external water pump with adjustable fluid velocity was used to provide experiments with varying fluid velocities.

Using signals from the high-precision, non-invasive ultrasonic flow meter, the water velocity was managed in a closed-loop control system [37]. Two strain gauges were positioned symmetrically on both sides of the water tunnel to assess the FSI. Both strain gauges were attached to the ground on one side and to a transparent plate (made of polycarbon) on the other. Ball bearings supported a polycarbonate plate to prevent friction.

A servomechanism (Dynamixel MX-12W http:/ / www.dynamixel.com/ (accessed on 7 December 2021)) was installed in the center of the polycarbonate plate for fin movement. The significant characteristics of the Dynamixel MX-12W are stall torque $(M)$ of $0.2 \mathrm{Nm}$ (at $12 \mathrm{~V}, 1.4 \mathrm{~A}$ ) and a top speed of $470 \mathrm{rpm}$ (revolutions per minute). This allowed the fins to be moved with oscillation motions of up to $7.8 \mathrm{~Hz}$ for unload movement. 
The force between the fin and the water was measured directly using different fins linked to the servomechanism. Furthermore, the control algorithm was used for the water velocity, fin frequency and amplitude with restrictions on the desired Strouhal number. Finally, the trailing edge's peak-to-peak amplitude was measured using the vision algorithm described in the authors' paper [41].

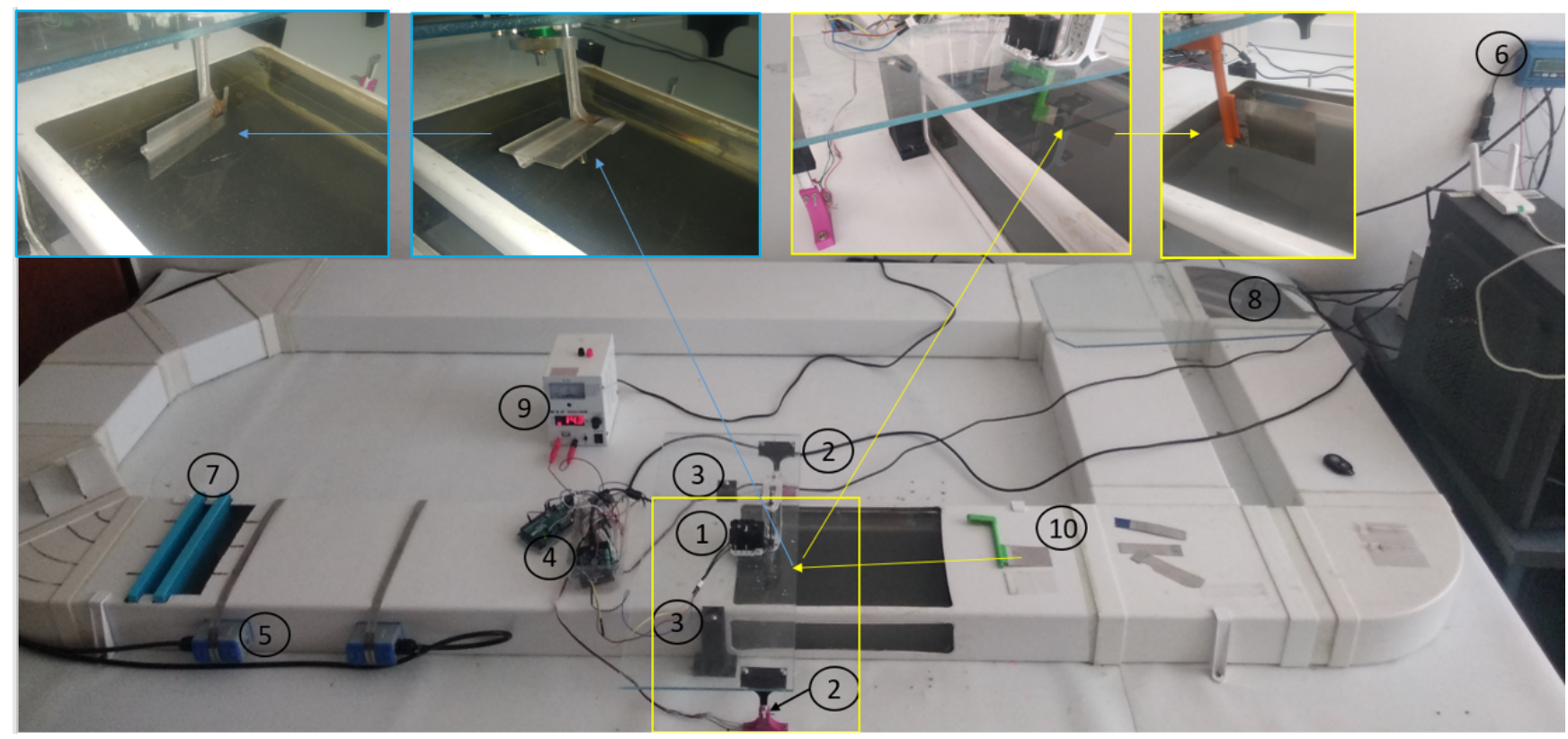

Figure 5. The laboratory water tunnel: 1-servomechanism Dynamixel MX-12W, 2—strain gauges, 3-ball bearings, 4micro-controller unit (MCU) and strain gauges amplifier, 5-ultrasonic flow sensors, 6-ultrasonic flow unit, 7-water steering wheels, 8-external water pump, 9-power supply, and 10-the fin.

\section{Results and Discussion}

For the proposed propulsion system, the example of the net thrust measurement result as a function of time with oscillation frequency $f=0.7 \mathrm{~Hz}$ is presented in Figure 6. On the left side of the figure, the position of the fin is presented during the power stroke (upper part) and during the recovery stroke (lower part). As can be seen in Figure 6, the measured value of FSI is characterized by fluctuations of the function of time. Therefore, the final values of the net thrust and frequency presented later in this paper were received by averaging over 10 periods of servomotor oscillations.

Both kinds of propulsion systems were tested for different frequencies of the fin oscillations and for different water velocities as well. The same frequency was used for each tested fin to limit the number of dependent variables. The mean value of the difference between the thrust and the drag force (the net thrust) was measured according to Formula (8) and is presented in (Figures 7-13).

Figures 7-9 show the series of mean values of the net thrust as a function of fin movement frequency for different water velocities. An external water pump was used to control the water velocity in the water tunnel to imitate FSI, as for the fin's forward speed in relation to the water. The water velocity varied between 0 and $20 \mathrm{~cm}$ per second $(u=0.2 \mathrm{~m} / \mathrm{s})$. The mean thrust for all frequencies was most significant when the water velocity was equal to zero $(u=0 \mathrm{~m} / \mathrm{s})$. 


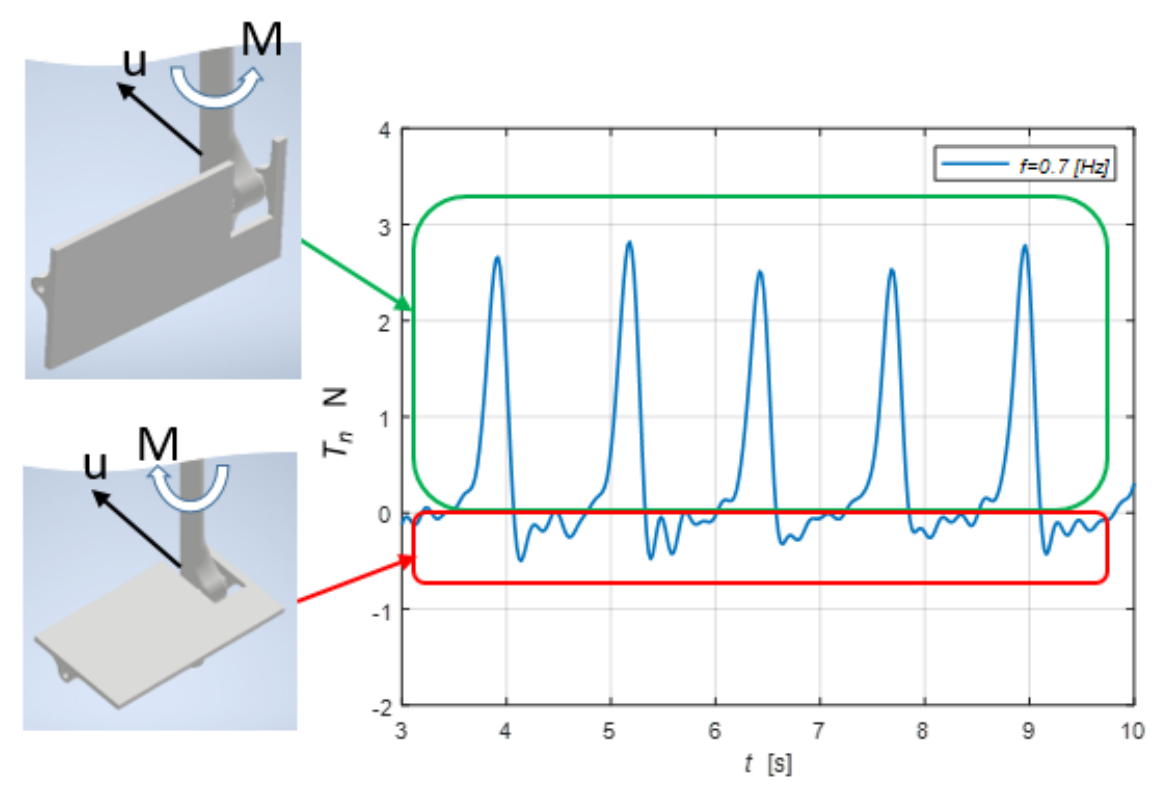

Figure 6. The net thrust as a function of time for an undulating frequency $f=0.7 \mathrm{~Hz}$.

For that measurement condition, the drag force was equal to zero for fish-like flapping foils. In contrast, for the fin with an additional joint, the thrust was much higher than the drag force. The mean net thrust for flexible polycarbonate fin movements at a frequency of $1 \mathrm{~Hz}$ was up to $40 \mathrm{mN}$. When increasing the frequency up to $4 \mathrm{~Hz}$, the net thrust increased up to $140 \mathrm{mN}$ (Figure 7). Raising the frequency further did not increase the mean net thrust.

Increasing the fluid's speed simulates the fin's movement against the fluid. When the velocity of the water increases, the tested fins must overcome fluid resistance. As a result, as the water velocity increases, and the average net thrust decreases. The nature of the changes in the mean net thrust is revealed by the approximation of the set of measurement points. It can also be seen that, for flexible fins (placed along the fluid stream) at low frequencies, the mean net thrust rapidly increases with increasing fin frequency, reaches a maximum, and then remains constant with further increases in fin frequency oscillation movement (Figures 7 and 8).

For fins with an additional axis of rotation, the mean thrust increases with respect to the fin oscillation frequency (Figure 9) according to Equation (9). This is because a higher fin oscillation frequency creates a faster fin velocity through the fluid. Hence, the driving force increases by the square of the speed.

Furthermore, during the return movement, the drag force is proportional to the speed, but the drag coefficient $\left(C_{f}\right)$ and frontal surface $(S(\alpha, \beta))$ decrease due to the rotation of the fin to $90^{\circ}$ provided by the additional joint. Furthermore, the acceleration can be adjusted so that, according to Equation (9), the forward acceleration can be greater than the return acceleration. 


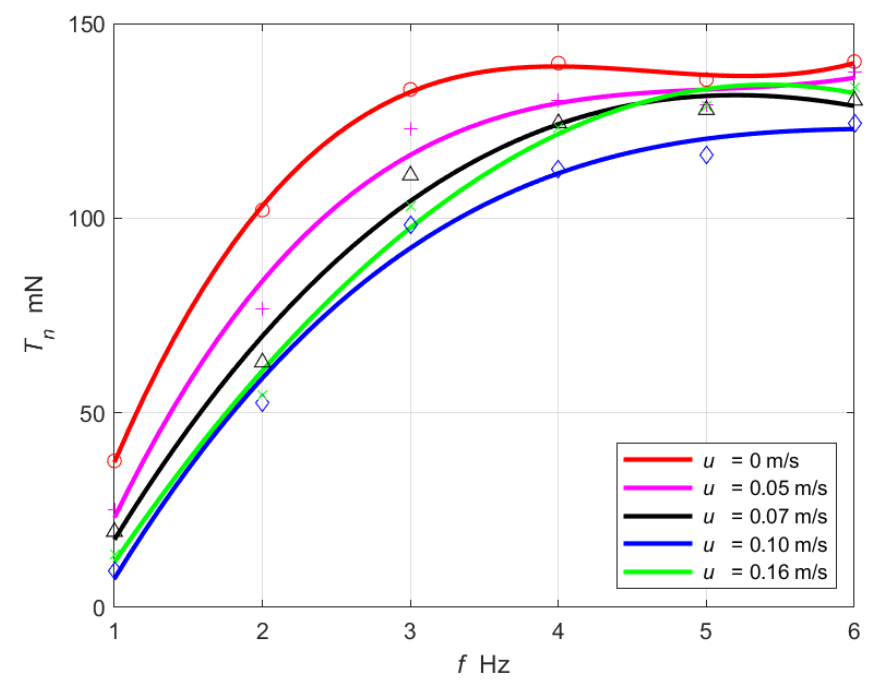

Figure 7. The mean value of the net thrust for a flexible fin made from polycarbonate.

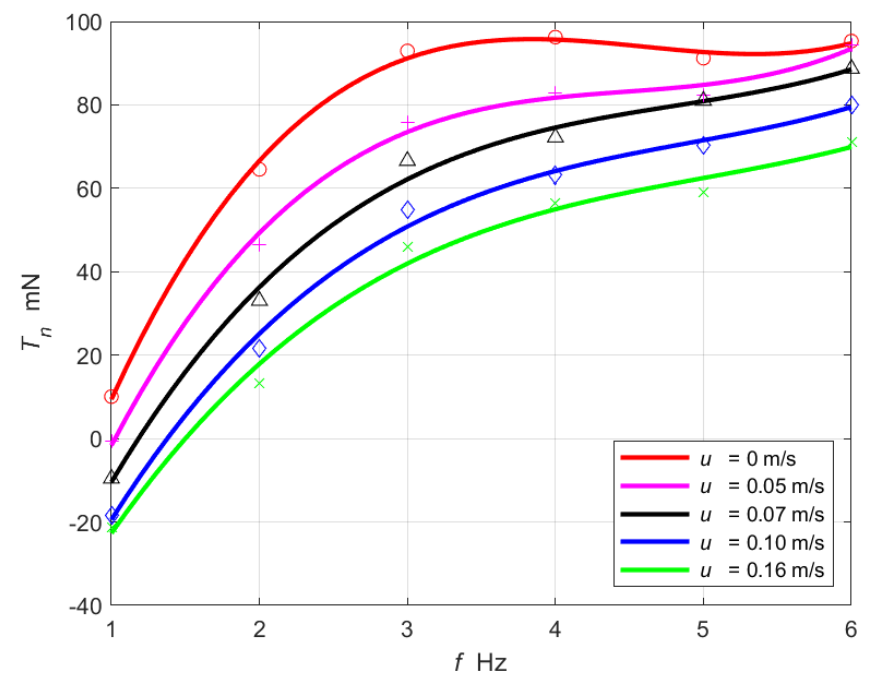

Figure 8. The mean value of the net thrust for a flexible fin made from a thin steel sheet.

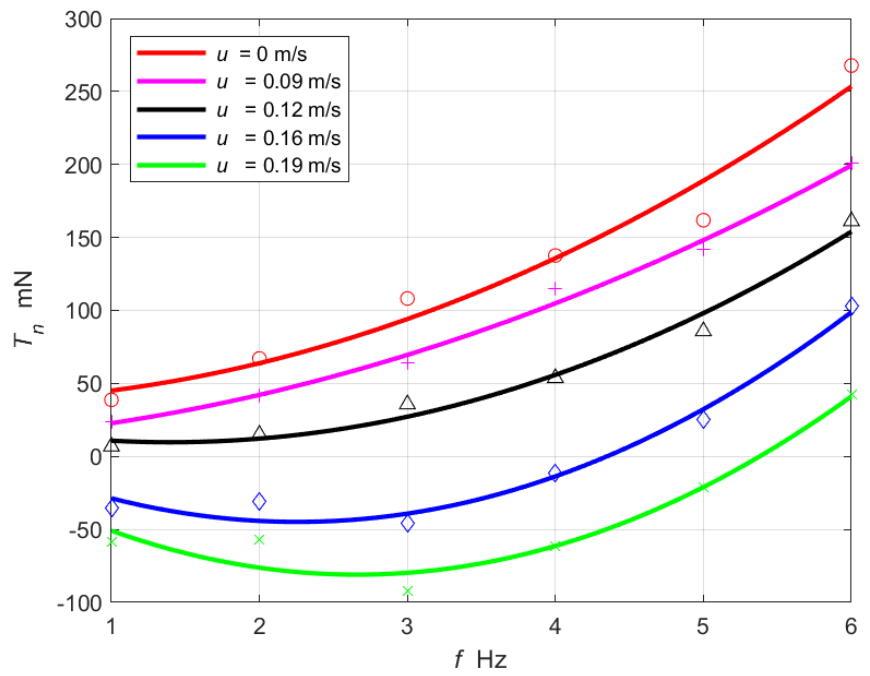

Figure 9. The mean value of the net thrust for an inflexible fin with an additional joint. 
Figure 10 shows the relationship between the net propulsive efficiency as a function of the Strouhal number. For varied fin frequencies, trailing edge amplitudes and fluid velocities, the same Strouhal number can be obtained. According to the Equation (3), as the frequency of the fin movement increases and the water speed increases, the Strouhal number may remain constant, but the net propulsive efficiency varies.

Taking into consideration the nature of the characteristic changes, it can be seen that the net propulsive efficiency increases due to the Stouhal number for the propulsion system with the additional joint. Figure 10 shows that flexible fins have a form comparable to the conventional efficiency curve, displaying efficiency as a function of the Strouhal number given in $[24,42]$.

The observed results are compatible with the literature research $[43,44]$, which revealed that the optimal efficiency [45] of the drive system for fish-like movements improves in the Strouhal number range of 0.25 to 0.35 [28].

Further, the stiffness [46] difference between the tested fish-like flapping foils (polycarbonate and steel fins) was responsible for the differences. During the investigation, there were different varieties of polycarbonate fins. Thus, the best one was chosen from a wide range of flexible parameters. There were not as many flexible fins available among thin sheet steel fins.

The net efficiency is zero when the mean net thrust is zero, which means that the thrust compensates for the drag force. If the thrust is lower than the drag force, then the net efficiency is negative. This means that the fluid velocity in the water tunnel is higher than the force with which the propulsion system would move forward in an unmovable fluid.

If the resistance of the investigated propulsion system were known, then the quasipropulsive efficiency defined in the paper [22] could be calculated. This could provide a more intuitive description of the efficiency, including the resistance. Then, there would not be a negative value of efficiency. For the tested propulsion system, the distinction between thrust and drag force cannot be made. The negative efficiency means that, at a constant water speed, the propulsion system cannot compensate for the drag to maintain a cruising velocity $(u)$.

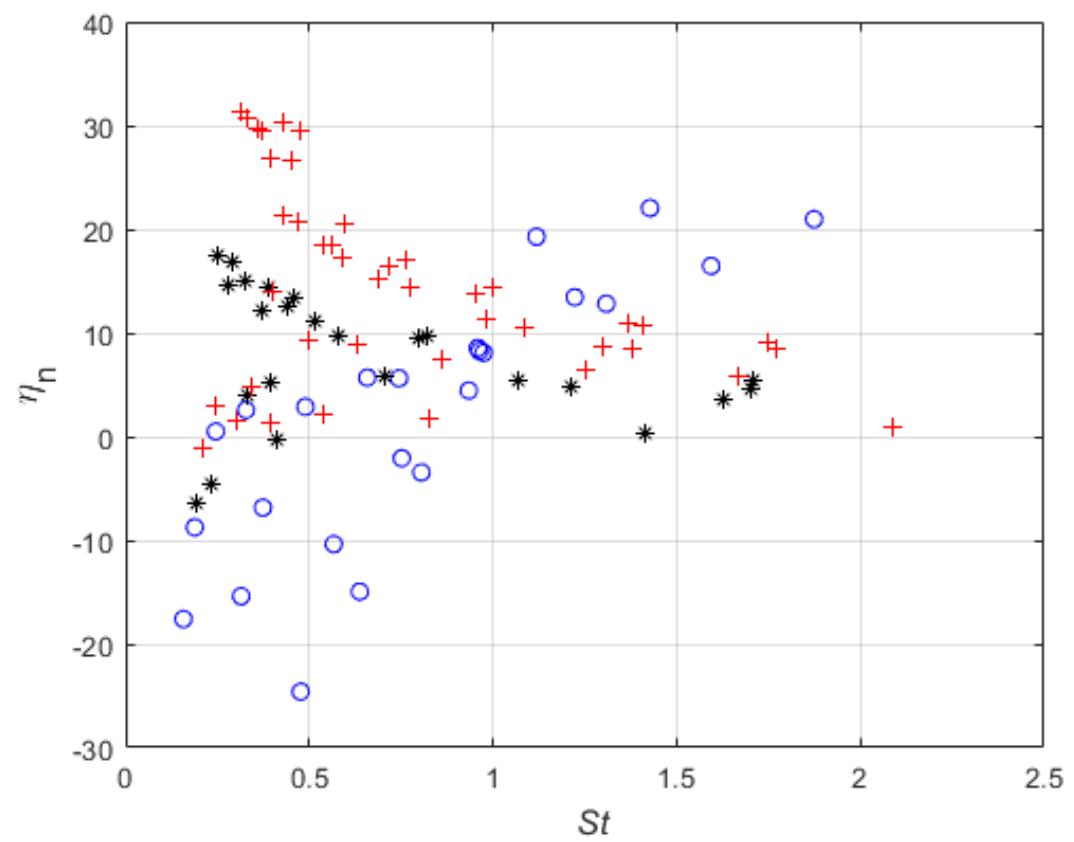

Figure 10. The net propulsive efficiency as a function of the Strouhal number for fins: + made from polycarbonate, o with an additional joint, and $*$ made from a thin steel sheet.

The design of the propulsion system with an additional joint achieved the best net efficiency for Strouhal numbers more significant than 1, indicating that the water velocity 
was equal to zero (Figure 11) or had a low value (Figure 12). This is analogous to fish that use a pectoral fin to swim at a slow speed but with high energy efficiency [47]. In addition, it is shown in Figures 11-13 that the net thrust for a propulsion system with an additional joint increases with higher frequency, according to the mathematical relationship in Equation (9).

For the results presented in Figure 11, where the water velocity is equal to zero, the thrust was measured for the drag force equal to zero. This indicates that, for that measurement condition, only the thrust is generated. It can be seen from the experimental measurements that the fin with an additional joint has an almost two-times higher thrust compared with the fish-like flapping foil propulsion system.

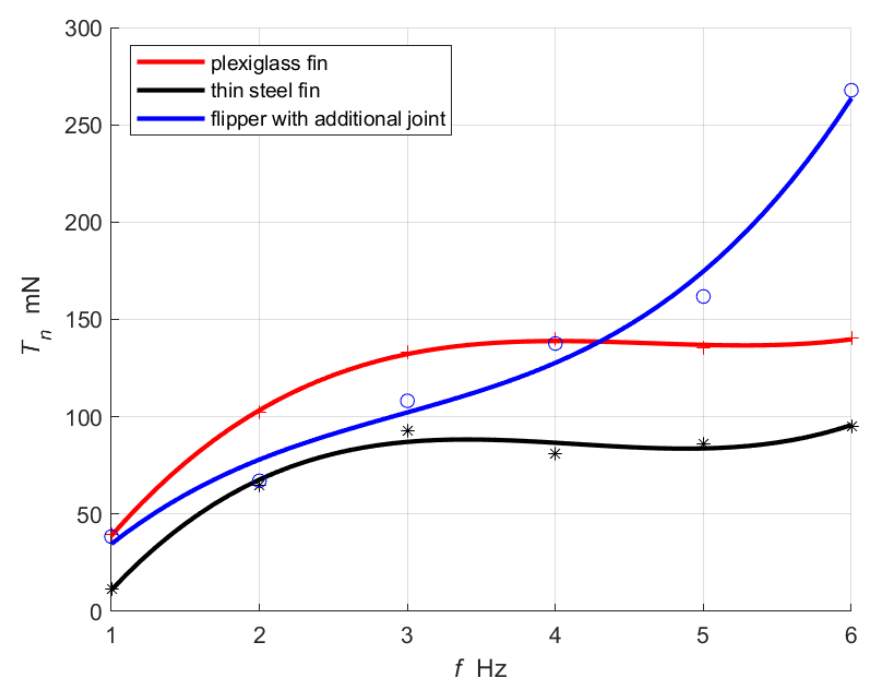

Figure 11. The mean thrust generated by fins for different frequencies and fluid velocity $u=0 \mathrm{~m} / \mathrm{s}$.

Figure 12 indicates the mean value of the net thrust, which is higher for the propulsion system with an additional joint in comparison to fish-like flapping foils in the frequency range above $4 \mathrm{~Hz}$. Furthermore, the proportional relationship between the thrust and fin frequency strengthens the BUUV's course control. When hydroacoustic sensors are utilised [48], this control condition is desirable for the BUV [49]. The measurements with a propulsion system implemented as a pectoral and/or caudal fin is to be the subject of future research.

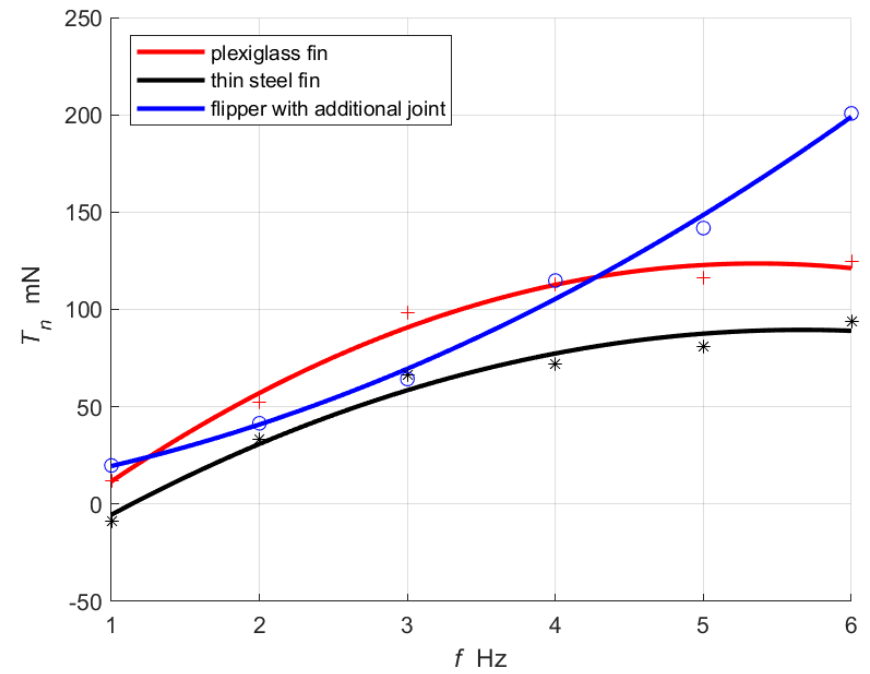

Figure 12. The mean net thrust generated by fins for different frequencies and fluid velocity $u=0.1 \mathrm{~m} / \mathrm{s}$. 
For the highest value of water velocity available in the water tunnel (Figure 13), the propulsion system with an additional joint had a lower value of the net thrust than both fish-like flapping foil propulsion systems. In addition, it is worth noting that, for water velocity $u=0.2 \mathrm{~m} / \mathrm{s}$, the net thrust force characteristics cross zero for all the tested fins. This gives information about the frequency needed to generate thrust to overcome the drag force and to swim with a constant speed. This also suggests further research on the propulsion system, which will cover the combination of flexible fins with an additional joint.

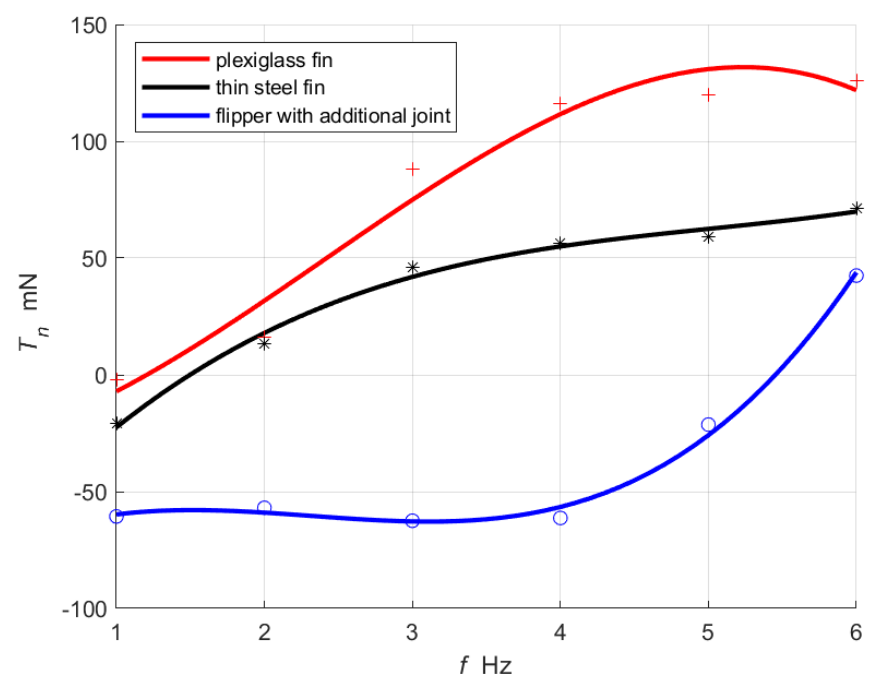

Figure 13. The mean net thrust generated by fins for different frequencies and fluid velocity $u=0.2 \mathrm{~m} / \mathrm{s}$.

\section{Conclusions and Future Work}

In the domains of bio-locomotion and biomimetics, the study of bodies moving in a fluid is an intriguing and challenging research topic. The number of design and control variables was kept low to demonstrate the difference between an innovative propulsion system and an undulating system. Increasing the velocity of the fins during thrust generation while decreasing the velocity of the fins during the returning movement should result in even better efficiency.

The fin velocity was determined by the specifications of the available servomotors and water tunnel dimensions. The proposed propulsion system design has higher thrust, and the higher net efficiency for low-speed movement ensures the biomimetic nature of the movement.

Future tests will be conducted, including the impact of the body on the propulsion system characteristics. The findings encourage further investigation into the effects of mass ratio, non-uniform stiffness and background turbulence on unsteady dynamics. Future research will concentrate on the Particle Image Velocimetry (PIV) approach, which will be used to conduct a more in-depth investigation of the FSI. This analysis method allows for a comparison of the created thrust to the speed of the water near the fins.

We will test a propulsion system with an additional joint and flexible fins as well as various movement styles. This can lead to a more accurate replica of a living marine organism with high energy efficiency. Finally, the promising results presented in the paper lead us to expect more efficient undulating propulsion for BUUVs in the near future.

\section{Patents}

On 6 December 2019, the propulsion system design named "Multi-fin wave thruster with resistance reduction in the return cycle, especially for a biomimetic underwater vehicle" was registered at the Patent Office of the Republic of Poland under the number P.432101. The patent application process is now underway. 
Author Contributions: Conceptualization, P.P. and P.S.; methodology, P.P.; software, P.S. and P.P.; validation, P.P.; formal analysis, P.P.; investigation, P.S., P.P., M.P.; resources, P.P.; data curation, P.S., P.P.; writing—original draft preparation, P.P.; writing—review and editing, P.S., K.N., K.J., M.Ż.; visualization, P.P., P.S.; supervision, P.S.; project administration, P.P.; funding acquisition, P.S. All authors have read and agreed to the published version of the manuscript.

Funding: The paper was financed by a Polish Ministry of Defense Research Grant titled “Model studies of the characteristics of an undulating propulsion system".

Institutional Review Board Statement: Not applicable.

Informed Consent Statement: Not applicable.

Data Availability Statement: Not applicable.

Conflicts of Interest: The authors declare no conflict of interest.

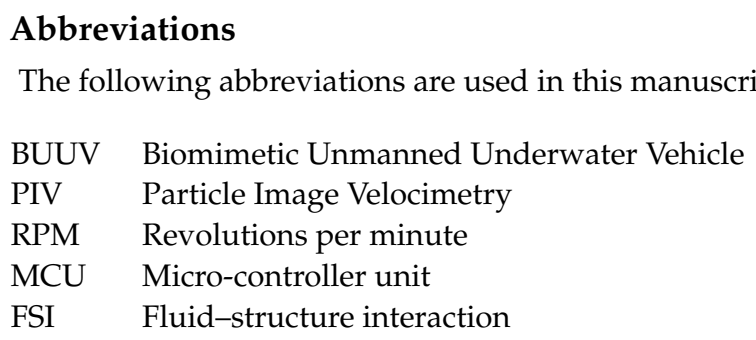

\section{References}

1. Mannam, N.P.B.; Krishnankutty, P. Biological Propulsion Systems for Ships and Underwater Vehicles. In Propulsion Systems; IntechOpen: London, UK, 2019; p. 113. [CrossRef]

2. Sitorus, P.E.; Nazaruddin, Y.Y.; Leksono, E.; Budiyono, A. Design and Implementation of Paired Pectoral Fins Locomotion of Labriform Fish Applied to a Fish Robot. J. Bionic Eng. 2009, 6, 37-45. [CrossRef]

3. Shi, L.; Guo, S.; Mao, S.; Yue, C.; Li, M.; Asaka, K. Development of an Amphibious Turtle-Inspired Spherical Mother Robot. J. Bionic Eng. 2013, 10, 446-455. [CrossRef]

4. Szymak, P.; Przybylski, M. Thrust measurement of biomimetic underwater vehicle with undulating propulsion. Sci. J. Pol. Nav. Acad. 2018, 213, 69-82. [CrossRef]

5. Salazar, R.; Quintana, R.; Abdelkefi, A. Role of Electromechanical Coupling, Locomotion Type and Damping on the Effectiveness of Fish-Like Robot Energy Harvesters. Energies 2021, 14, 693. [CrossRef]

6. Marcin, M.; Adam, S.; Jerzy, Z.; Marcin, M. Fish-like shaped robot for underwater surveillance and reconnaissance-Hull design and study of drag and noise. Ocean Eng. 2020, 217, 107889. [CrossRef]

7. Szymak, P.; Malec, M.; Morawski, M. Directions of development of underwater vehicle with undulating propulsion. Pol. J. Environ. Stud. Hard Publ. Co. 2010, 19, 107-110.

8. Piskur, P.; Gasiorowski, M. Digital Signal Processing for Hydroacoustic System in Biomimetic Underwater Vehicle. NASE MORE 2020, 67, 14-18.10.17818/NM/2020/1.3. [CrossRef]

9. Tytell, E.D.; Hsu, C.Y.; Williams, T.L.; Cohen, A.H.; Fauci, L.J. Interactions between internal forces, body stiffness, and fluid environment in a neuromechanical model of lamprey swimming. Proc. Natl. Acad. Sci. USA 2010, 107, 19832-19837. [CrossRef] [PubMed]

10. Grzadziela, A.; Szymak, P.; Piskur, P. Method for assessing the dynamics and efficiency of diving fins. Acta Bioeng. Biomech. 2020, 22, 139-150.[CrossRef]

11. Blondeaux, P.; Fornarelli, F.; Guglielmini, L.; Triantafyllou, M.; Verzicco, R. Vortex structures generated by a finite-span oscillating foil. In Proceedings of the 43rd AIAA Aerospace Sciences Meeting and Exhibit, Reno, NV, USA,10-13 January 2005; p. 84. [CrossRef]

12. Morawski, M.; Malec, M.; Szymak, P.; Trzmiel, A. Analysis of Parameters of Traveling Wave Impact on the Speed of Biomimetic Underwater Vehicle. In Solid State Phenomena; Trans Tech Publications Ltd.: Zürich, Switzerland, 2014, Volume 210, pp. 273-279.

13. Michelin, S.; Llewellyn Smith, S.G. Resonance and propulsion performance of a heaving flexible wing. Phys. Fluids 2009, 21, 071902. [CrossRef]

14. Hozyn, S. An Automated System for Analysing Swim-Fins Efficiency. NASE MORE 2020, 67, 10-17. [CrossRef]

15. Szymak, P.; Praczyk, T.; Naus, K.; Szturomski, B.; Malec, M.; Morawski, M. Research on biomimetic underwater vehicles for underwater ISR. In Proceedings of the International Society for Optics and Photonics, Baltimore, MD, USA, 17-21 April 2016; Volume 9831, p. 98310L. [CrossRef]

16. Piskur, P.; Szymak, P.; Jaskólski, K.; Flis, L.; Gąsiorowski, M. Hydroacoustic System in a Biomimetic Underwater Vehicle to Avoid Collision with Vessels with Low-Speed Propellers in a Controlled Environment. Sensors 2020, 20, 968. [CrossRef] 
17. Singh, N.; Gupta, A.; Mukherjee, S. A dynamic model for underwater robotic fish with a servo actuated pectoral fin. SN Appl. Sci. 2019, 1, 1-9. [CrossRef]

18. Li, Z.; Xu, W.; Wang, W.; Du, Y.J. Dynamic modeling and experimental research on the linear swimming of a biomimetic cod fish driven by pectoral fin with two degrees of freedom and flexible body. J. Ship. Mech. 2017, 21, 513-527.

19. Nguyen, T.T.; Lee, B.R.; Vo, T.Q. Dynamic analysis of a robotic fish propelled by flexible folding pectoral fins. Robotica 2020 38, 699-718. [CrossRef]

20. Behbahani, S.B.; Tan, X. Bio-inspired flexible joints with passive feathering for robotic fish pectoral fins. Bioinspir. Biomimet. 2016, 11, 036009. [CrossRef]

21. Piskur, P.; Szymak, P.; Flis, L.; Sznajder, J. Analysis of a Fin Drag Force in a Biomimetic Underwater Vehicle. NAŠE MORE Znan. časopis Za More I Pomor. 2020, 67, 192-198. [CrossRef]

22. Maertens, A.P.; Triantafyllou, M.S.; Yue, D.K.P. Efficiency of fish propulsion. Bioinspir. Biomimet. 2015, 10, 046013. [CrossRef]

23. Collins, K.M. The Effects of Asymmetry on Oscillatory Propulsion. Ph.D. Thesis, University of Bath, Bath, UK, 2012.

24. Floryan, D.; Van Buren, T.; Smits, A.J. Efficient cruising for swimming and flying animals is dictated by fluid drag. Proc. Natl. Acad. Sci. USA 2018, 115, 8116-8118. [CrossRef]

25. Wu, X.; Zhang, X.; Tian, X.; Li, X.; Lu, W. A review on fluid dynamics of flapping foils. Ocean Eng. 2020, 195, 106712. [CrossRef]

26. Buckingham, E. The principle of similitude. Nature 1915, 96, 396-397. [CrossRef]

27. Liu, W.; Xiao, Q.; Cheng, F. A bio-inspired study on tidal energy extraction with flexible flapping wings. Bioinspir. Biomimet. 2013, 8, 036011. [CrossRef]

28. Triantafyllou, G.S.; Triantafyllou, M.S.; Grosenbaugh, M.A. Optimal thrust development in oscillating foils with application to fish propulsion. J. Fluids Struct. 1993, 7, 205-224. [CrossRef]

29. Baik, Y.S.; Bernal, L.P. Experimental study of pitching and plunging airfoils at low Reynolds numbers. Exp. Fluids 2012, 53, 1979-1992. [CrossRef]

30. Luo, Y.; Xiao, Q.; Shi, G.; Pan, G.; Chen, D. The effect of variable stiffness of tuna-like fish body and fin on swimming performance. Bioinspir. Biomimet. 2020, 16, 016003. [CrossRef] [PubMed]

31. Piskur, P.; Szymak, P.; Kitowski, Z.; Flis, L. Influence of fin's material capabilities on the propulsion system of biomimetic underwater vehicle. Polish Marit. Res. 2020, 4, 179-185. [CrossRef]

32. Bevilaqua, P.; Yam, C. Propulsive Efficiency of Wake Ingestion. J. Propuls. Power 2020, 36, 517-526. [CrossRef]

33. Gough, W.T.; Smith, H.J.; Savoca, M.S.; Czapanskiy, M.F.; Fish, F.E.; Potvin, J.; Bierlich, K.; Cade, D.E.; Clemente, J.D.; Kennedy, J.; others. Scaling of oscillatory kinematics and Froude efficiency in baleen whales. J. Exp. Biol. 2021. [CrossRef] [PubMed]

34. Heller, V. 8.04-Development of Wave Devices from Initial Conception to Commercial Demonstration. In Comprehensive Renewable Energy; Sayigh, A., Ed.; Elsevier: Oxford, UK, 2012; pp. 79-110. [CrossRef]

35. Rodič, M.; Milanovič, M.; Truntič, M. Digital Control of an Interleaving Operated Buck-Boost Synchronous Converter Used in a Low-Cost Testing System for an Automotive Powertrain. Energies 2018, 11, 2290. [CrossRef]

36. De Santis, M.; Agnelli, S.; Patanè, F.; Giannini, O.; Bella, G. Experimental Study for the Assessment of the Measurement Uncertainty Associated with Electric Powertrain Efficiency Using the Back-to-Back Direct Method. Energies 2018, 11, 3536. [CrossRef]

37. Piskur, P.; Szymak, P.; Sznajder, J. Identification in a laboratory tunnel to control fluid velocity. In Advanced, Contemporary Control; Springer: Berlin/Heidelberg, Germany, 2020; pp. 1543-1552.

38. Liu, G.; Dong, H. Effects of tail geometries on the performance and wake pattern in flapping propulsion. In Proceedings of the ASME 2016 Fluids Engineering Division Summer Meeting collocated with the ASME 2016 Heat Transfer Summer Conference and the ASME 2016 14th International Conference on Nanochannels, Microchannels, and Minichannels, Washington, DC, USA, 10-14 July 2016; Volume 50299, p. V01BT30A002. [CrossRef]

39. Engels, T.; Kolomenskiy, D.; Schneider, K.; Sesterhenn, J. Numerical simulation of vortex-induced drag of elastic swimmer models. Theor. Appl. Mech. Lett. 2017, 7, 280-285. [CrossRef]

40. Szturomski, B.; Kiciński, R. Material Properties of HY 80 Steel after 55 Years of Operation for FEM Applications. Materials 2021, 14, 4213. [CrossRef]

41. Jurczyk, K.; Piskur, P.; Szymak, P. Parameters identification of the flexible fin kinematics model using vision and Genetic Algorithms. Polish Marit. Res. 2020, 2, 39-47. [CrossRef]

42. Ebrahimi, M.; Abbaspour, M. A comparative numerical study on the performances and vortical patterns of two bioinspired oscillatory mechanisms: Undulating and pure heaving. Appl. Bionics Biomech. 2015, 2015, 325934. [CrossRef]

43. Link, O.; Sanhueza, C.; Arriagada, P.; Brevis, W.; Laborde, A.; González, A.; Wilkes, M.; Habit, E. The fish Strouhal number as a criterion for hydraulic fishway design. Ecol. Eng. 2017, 103, 118-126. [CrossRef]

44. Dabiri, J.O. Optimal vortex formation as a unifying principle in biological propulsion. Annu. Rev. Fluid Mech. 2009, 41, 17-33. [CrossRef]

45. Eloy, C. Optimal Strouhal number for swimming animals. J. Fluids Struct. 2012, 30, 205-218. [CrossRef]

46. Riggs, P.; Bowyer, A.; Vincent, J. Advantages of a biomimetic stiffness profile in pitching flexible fin propulsion. J. Bionic Eng. 2010, 7, 113-119. [CrossRef]

47. Taylor, G.K.; Nudds, R.L.; Thomas, A.L. Flying and swimming animals cruise at a Strouhal number tuned for high power efficiency. Nature 2003, 425, 707-711. [CrossRef] 
48. Piskur, P.; Szymak, P. Digital Signal Processing for Hydroacoustic Passive Obstacle Detection System in Biomimetic Underwater Vehicle. In Proceedings of the 2018 International Conference on Applied Mathematics \& Computational Science (ICAMCS.NET), Budapest, Hungary, 6-8 October 2018; pp. 110-114. [CrossRef]

49. Cheong, S.; Kim, Y.J.; Chun, J.H.; Kim, J.K.; Huh, S. Integrated Offshore Seismic Survey Using an Unmanned Wave Glider. Energies 2021, 14, 297. [CrossRef] 